\title{
Prediction of Random Errors in Sound Intensity Measurement
}

\author{
Finn Jacobsen ${ }^{\dagger}$ \\ Department of Acoustic Technology, Technical University of Denmark, \\ Building 352, Ørsteds Plads, DK-2800 Lyngby, Denmark
}

(Received 16 May 2000; accepted 15 September 2000)

\begin{abstract}
It is well known that the random errors in sound intensity estimates can be much larger than the theoretical minimum value determined by the BT-product, in particular under reverberant conditions, in the near field of structural sources, and when there are several independent sources present. More than ten years ago it was shown that one can predict the random errors in estimates of the sound intensity in, say, one-third octave bands from the power and cross power spectra of the two signals from a sound intensity probe, determined with fine spectral resolution with a dual channel FFT analyser. This is not very practical, though. In this paper it is demonstrated that one can predict the random errors from the power and cross power spectra determined with the same spectral resolution as the sound intensity itself.
\end{abstract}

${ }^{\dagger}$ Member of the International Institute of Acoustics and Vibration (IIAV)

\section{INTRODUCTION}

Random errors due to incomplete time averaging reveal themselves by poor repeatability. Such errors can be of concern in several applications of sound intensity measurements. The averaging time needed to ensure a given accuracy depends very much on the local properties of the sound field, and some positions will require a very long averaging time. However, it is obviously inconvenient to use a very long averaging time at each point if the sound intensity is to be mapped in front of a large, complicated source of noise. In fact, even if an automated measurement system is available, the averaging time may be of concern, for example because the sound source under study is not completely stable over a long period of time.

That the normalised random errors in sound intensity measurements can be much larger than the theoretical minimum value of $1 / \sqrt{B T}$ known from mean square estimation, where $B$ is the bandwidth and $T$ is the averaging time, was pointed out by van Zyl and Anderson as early as $1975 .{ }^{1}$ Some years later Seybert ${ }^{2}$ and Pascal $^{3}$ derived theoretical expressions that showed that the random errors can be very large when the phase angle between two pressure signals from the sound intensity probe is small and the coherence of the signals is less than unity. (Both Seybert and Pascal assumed that the sound intensity was measured with the usual $p-p$ technique using an intensity probe based on two closely spaced pressure microphones. ${ }^{4}$ ) In 1983 Dyrlund $^{5}$ made an interesting analysis of three simple cases on the basis of Seybert's expression. One of Dyrlund's observations was that, given a certain structure of the sound field, the random error depends essentially on the pressure-intensity index of the sound field and is practically independent of the frequency and the distance between the two microphones of the sound intensity probe. The results of Seybert, Pascal and Dyrlund had indicated the possibility of very large random errors, and in the following years several authors expressed concern about this source of error. ${ }^{6-9}$
However, the expressions derived by Seybert and Pascal are extremely sensitive to resolution bias errors in the estimated coherence, as pointed out by Watkinson, who also remarked that 'the random errors can be grossly overestimated' if there are bias errors in the estimated coherence. ${ }^{10}$ In 1989 Jacobsen showed that although the expressions by Seybert and Pascal are correct in theory, they are often misleading in practice because it has been tacitly assumed that the sound intensity is determined with infinitely fine spectral resolution. ${ }^{11}$ For example, Jacobsen showed that the random errors can be very large even when the true coherence is unity, in disagreement with Seybert and Pascal's results. He also showed how one can predict the random errors if the power and cross power spectra of the two signals from the sound intensity probe are measured with fine spectral resolution.

Some of Dyrlund's experimental results had seemed to indicate that a biased estimate of the coherence function can be used in predicting random errors, ${ }^{5}$ in obvious conflict with Watkinson's considerations ${ }^{\mathbf{1 0}}$ and apparent disagreement with Jacobsen's theoretical results. ${ }^{11}$ More recent theoretical work seems to support Dyrlund's results. ${ }^{12,13}$ If the biased coherence can be used in estimating random errors then it would seem to be possible to predict such errors and thus the necessary averaging time in sound intensity measurements with ordinary filter bank analysers.

The purpose of this paper is to examine the matter.

\section{OUTLINE OF THEORY}

\subsection{General Formulations}

Seybert's expression for the normalised random error in sound intensity estimates determined with an FFT analyser has the form ${ }^{2}$

$$
\varepsilon\left\{\hat{I}_{r}(\omega)\right\}=\frac{\left[1 / \gamma_{12}^{2}(\omega)+\cot ^{2}(\varphi(\omega))\left(1-\gamma_{12}^{2}(\omega)\right) / 2 \gamma_{12}^{2}\right]^{1 / 2}}{\sqrt{n}}
$$

